\title{
Generation of small mono-disperse bubbles in axisymmetric T-junction: the role of swirl
}

\author{
Miguel A. Herrada, Alfonso M. Gañán-Calvo ${ }^{\dagger}$, José M. López-Herrera \\ Escuela Superior de Ingenieros, Universidad de Sevilla, \\ Camino de los Descubrimientos s/n, 41092, Spain
}

\begin{abstract}
The dynamics of micro-bubble formation in an axisymmetric T-junction for a gas-liquid system is analyzed. The approach adopted involves the creation of a tapering gas-liquid meniscus from which a steady gas ligament issues by the introduction of a coaxial swirl in the liquid stream. A simple and easy geometry (an axisymmetric T-Junction) suffices to introduce the swirl and to stabilize the meniscus, leading to the formation of small monodisperse bubbles. Full three-dimensional simulations (3D) have also been conducted to show that, even when the liquid injection is not perfectly axisymmetric, the bubbles generated under conditions of some focusing swirl are distinctively smaller than bubbles created in the absence of swirl. In such cases, the bubbles, produced at the trail of the vortex axis, become a serendipitous tool to visualize the non-axisymmetrical behavior of the vortex core, as shown by the simulations.
\end{abstract}




\section{†Corresponding author}

\section{INTRODUCTION}

The vast variety of potential applications (see for example Whitesides ${ }^{1}$ and Christopher and Anna $^{2}$ ) of a narrowly-distributed micron/nano sized bubble population has spurred the study on bubble production methods in the last decade. The most direct method for generating bubbles is the direct injection of a gas flow trough a nozzle (orifice or tube) into a quiescent liquid reservoir ${ }^{3}$. This direct injection results, in general, in a low frequency production of large bubbles. If a stable stream of equally sized small bubbles is desired, more sophisticated methods involving microfluidic devices are to be used. In such devices, the two-phase flow is usually generated by mechanical means using either a cross-flowing, a co-flowing or a flow focusing configuration ${ }^{2}$.

The most extended cross-flowing microfluidic device involves a T-Junction of capillary tubes (channels) of similar diameter (width) ${ }^{4,5}$. In this configuration the flowing liquid causes the capillary breakup of the cross-flowing gas arriving to the junction. As a result, the emerging bubbles may fill either a part of, or the complete duct cross section (unconfined bubble vs. slug flow). However, in both cases, the technical aim is the introduction of a quasi-periodic sequence of gas-liquid plugs into a downstream quiescent reservoir, the size of the bubbles being influenced by the length of the junction branches ${ }^{5-7}$.

In co-flowing setups the continuum liquid phase flows parallel to a duct through which the gas is injected ${ }^{8}$. In this configuration, bubbles are usually smaller when the liquid continuum phase is faster due to larger shear stress exerted on the interface. Bubble size scales with the inner diameter of the injection duct ${ }^{9}$.

Focusing the gas finger may also be achieved by forcing the liquid phase to cross a throttle orifice, either circular or elongated, which is located in front of the gas exit ${ }^{10}$; this is the so-called flow focusing configuration (Figure 1a). The flow focusing approach has demonstrated great flexibility as a means to control size, volume fraction and structure of the emulsions in a wide range of regimes and geometries under which this system can be

operated. Axisymmetric geometries where the first to be explored in the laboratory ${ }^{10}$, but soon after, planar geometries were experimented with success ${ }^{11,12}$. In particular, a specific planar geometry using a Hele-Shaw-like cell has been also proposed ${ }^{13}$ (Figure 1b). 
Conventional cross-flowing devices produce bubbles whose size is commonly of the order of a characteristic geometric size of the device; this is a consequence of the pinch-off process, which is strongly influenced by the smallest geometric feature of the device, i.e. the constriction orifice or the junction geometry. Only by using flow focusing or co-flowing would it be possible to obtain bubbles whose size is significantly smaller than the characteristic geometric length. Note, however, that in the case of a co-flowing setup, tip-streaming is only observed when the operating conditions are adjusted to produce a sufficiently high flow rate of the continuum liquid phase, while at the same time making sure that it flows in the Stokes regime around the gas source, two contradictory requirements. In contrast, less stringent conditions are required if the flow focusing configuration is used ${ }^{10}$, where relatively large Reynolds numbers for the liquid flow through the discharge orifice can be used. However, in spite of the strong pressure gradient associated to the abrupt discharge through the orifice (the pressure gradient leading to a substantial stabilization of the gas-liquid cusp-like meniscus), the small viscosity of water and its relatively large surface tension hampers the production of monodisperse microbubbles smaller than the discharge orifice. Indeed, we are unaware of any reported experiment where monodisperse microbubbles in water (in the absence of surfactants) are produced by a flow focusing configuration.

Recently, Herrada and Gañán-Calvo ${ }^{14}$ have proposed an axisymmetric flow focusing configuration in a new operation regime for the production of micro bubbles labeled as Swirl Flow Focusing method (SFF). This method is based on the superposition of a swirl component to the axisymmetric meridional flow of the continuum phase. The centrifugal forces associated to the swirl create an intense pressure gradient in the radial direction which stabilizes the gas tapering meniscus and helps focusing the gas finger into a short steady gas ligament. Using this method, using water as a focusing fluid, a stream of bubbles of a notably smaller size than the geometric characteristic length can be obtained.

In this paper, we aim to shed light on the experimental observations illustrated in Fig. $1(\mathrm{c})$ and $(\mathrm{d})$, where a very simple and promising geometry formed by just two silica capillaries aligned and slightly offset ${ }^{16}$ was sufficient to promote the conditions to generate a stream of extremely small monodisperse bubbles using water. In a first attempt to tackle the problem, we noticed that a detailed monitoring of the flow conditions at the gap between the end of the tubes, including inlet and outset, was very difficult to attain for this geometry [see Fig. $1(\mathrm{c})$ and $(\mathrm{d})]$, and the possible presence of swirl was impossible to assess experimentally. 

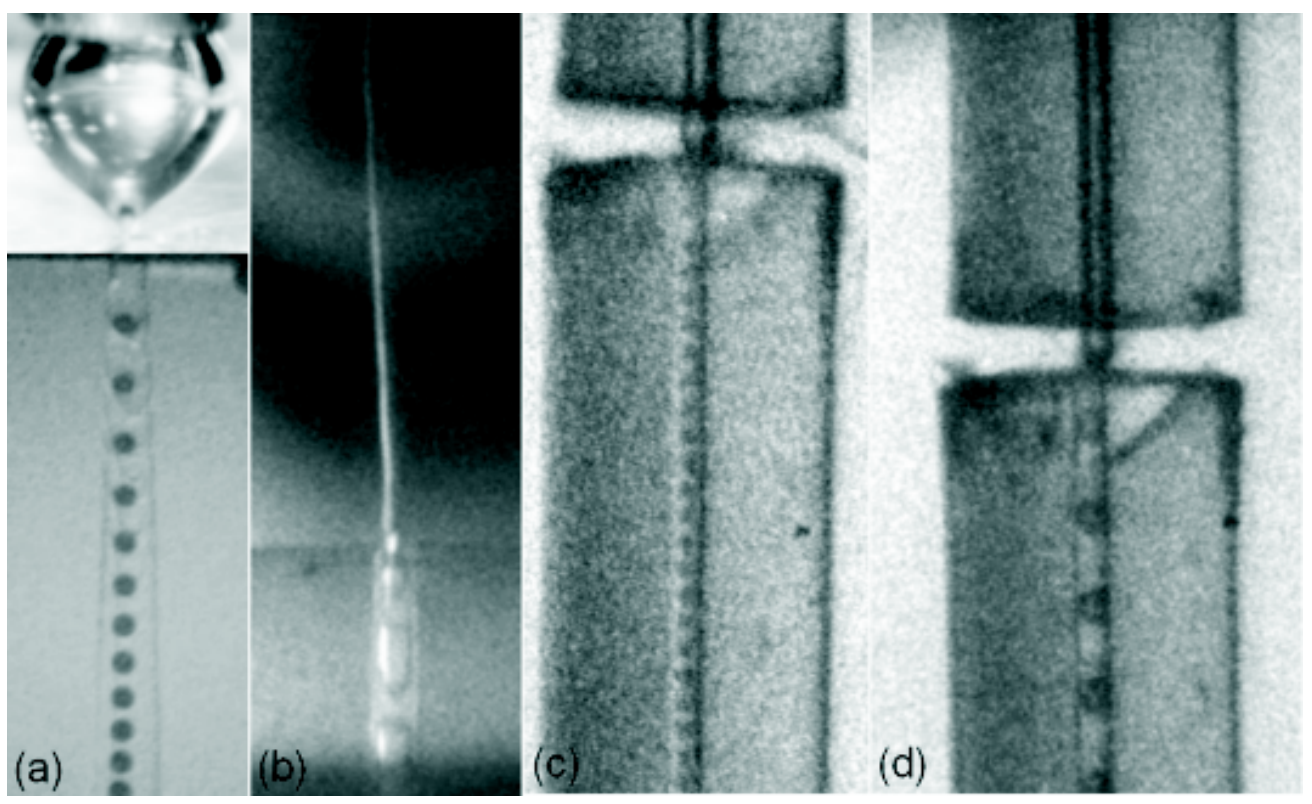

FIG. 1: (a) The axisymmetric flow-focusing configuration ${ }^{10}$. (b) The planar, Hele-Shaw-like cell configuration $^{13}$. (c)-(d) Two differently operated tube-tube geometries, forming an axisymmetric T-junction: in (c) the geometrical irregularities in the transversal cut of the silica tubes (O.D. 365 $\mu \mathrm{m}$, I.D. $40 \mu \mathrm{m}$ ) or the presence of some debris in the gap are believed to have led to the natural occurrence of swirl, and the production of very small bubbles, as numerically demonstrated in this work; in (d), the flow regime leads to gas dripping and plug-like bubbles (the swirl, if there is any, is not sufficient to avoid meniscus instability and dripping).

Thus, we decided to gain insight from a totally different perspective using full numerical simulation. As a step forward from the conclusions obtained by Herrada and Gañán-Calvo ${ }^{14}$, first we found that a smooth converging nozzle may be replaced by a simpler geometry (an axisymmetric T-Junction) with similar results. Second, using 3D numerical schemes, we explored whether the bubbling process is sensitive to azimuthal finite perturbations; indeed, swirling flows can experience helicoidal instabilities which are not present in axisymmetric simulations ${ }^{15}$. Last, this paper aims to provide a general physical layout of the production of very small bubbles using notably simple and robust means. 


\section{GOVERNING EQUATIONS AND BOUNDARY CONDITIONS}

The axisymmetric T-junction device and the computational domain used in this work are sketched in Fig. 2. A constant gas flow rate $Q_{g}$, flowing through a capillary tube of radius $R$, is discharged into a coaxial disk-like gap (radius $R_{1}$ and thickness $H$ ). The focusing liquid is injected with a flow rate $Q_{l}$ through this disk-like gap in the inward direction. Finally, the two-phase flow is drawn through an evacuation tube of identical radius $R$ and length $L_{1}$, aligned with the gas tube.

Due to the relatively small pressure variations used, for the purpose of this work and for simplicity of formulation the gas is assumed incompressible. Therefore, incompressible, axisymmetric, and unsteady Navier-Stokes equations in cylindrical $(z, r, \theta)$ coordinates are used to describe the time evolution of both fluids. The velocity and pressure fields are identified as $\mathbf{u}=(w, u, v)$ and $p$, respectively, and the subindexes $g$ and $l$ stand for gas and liquid. In addition, the 3D evolution of the flow will be analyzed by solving the N-S equation in cartesian $(z, x, y)$ coordinates $z=z, x=r \cos \theta$ and $y=r \sin \theta$.

Figure 2 shows the boundary conditions of the problem: (a) at the gas inlet, $z=0$, a Hagen-Poiseuille flow, $w_{g}(r, z=0, \theta)=W_{1}\left[1-(r / R)^{2}\right]$ and $v_{g}=0$, is specified; (b) at the liquid inlet, $r=R_{1}, L<z<L+H$, we assume that the inward radial velocity profile may fluctuate in the $\theta$-direction, $u_{l}\left(r=R_{1}, z, \theta\right)=-U_{2}(1+\Delta U \cos \theta)$ while the azimuthal velocity has an uniform profile $v_{l}\left(r=R_{1}, z, \theta\right)=\Gamma / R_{1}$, where $\Gamma$ is the imposed circulation; and (c) at the evacuation tube exit, $z=z_{\text {out }}=L+H+L_{1}$, outflow conditions, $\partial \mathbf{u} / \partial z=0$, are considered. Naturally, we impose non-slip condition in all solid walls and symmetry condition at the axis $r=0$ (only needed for the $2 \mathrm{D}$ simulations). It should be said that in the present computation, the amplitude of a perturbation imposed in the $\theta$-direction is not necessarily small, $0 \leq \Delta U \leq 1$.

Note that the corresponding gas and liquid flow rates can be derived from the inlet velocity field:

$$
\begin{aligned}
Q_{g} & =\int_{0}^{R} 2 \pi r w_{g}(r, z=0, \theta) \mathrm{d} r \\
Q_{l} & =\int_{L}^{L+H} \int_{0}^{2 \pi}-R_{1} u_{l}\left(r=R_{1}, z, \theta\right) \mathrm{d} \theta \mathrm{d} z .
\end{aligned}
$$

Parametric studies of the dimensionless variables involved are carried out next. The velocity field $\mathbf{u}$, lengths, time $t$, and pressure $p$ are scaled with the mean axial liquid velocity 


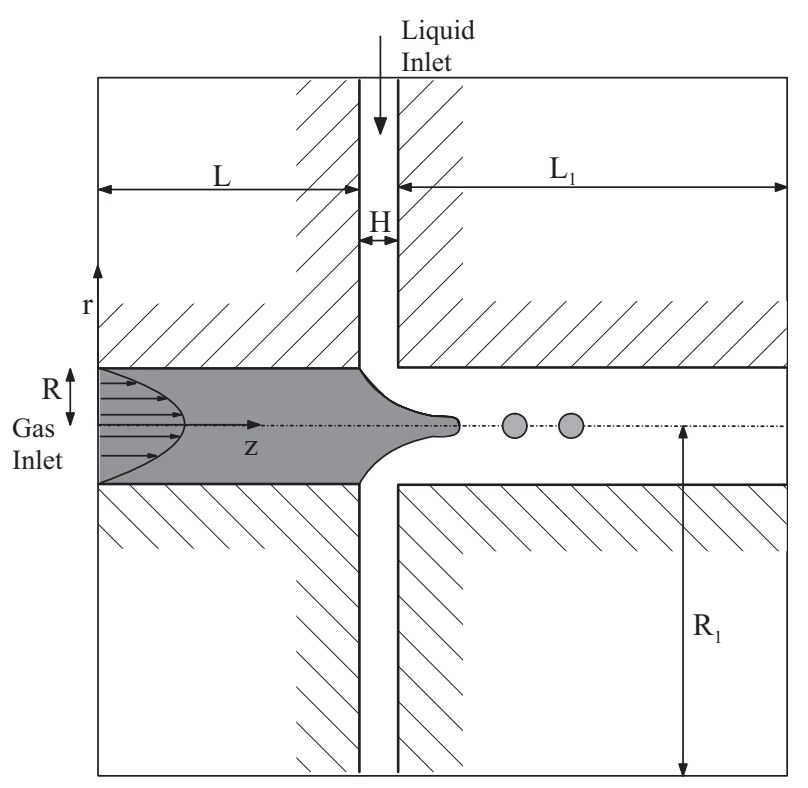

FIG. 2: Simulated boundary geometry and fluid flow domains.

at the pipe $W=Q_{l} /\left(\pi R^{2}\right)$, the discharge tube radius $R, R / W$, and $\rho_{l} W^{2}$, respectively, where $\rho_{l}$ is the density of the focusing liquid. In this work, a single geometry is considered, characterized by the following aspect ratios: $R_{1} / R=2.5, H / R=0.75, L / R=2.5$, and $L_{1} / R=11.75$. We have chosen a gas-liquid combination where

$$
\frac{\rho_{l}}{\rho_{g}}=833.33, \frac{\mu_{l}}{\mu_{g}}=55.55,
$$

$\rho_{l}\left(\rho_{g}\right)$ and $\mu_{l}\left(\mu_{g}\right)$ being the density and viscosity of the liquid (gas). This choice corresponds to an air stream drawn by a co-flowing water stream. The problem is governed by a set of five non-dimensional numbers as follows.

- The Reynolds and Weber numbers defined as

$$
\begin{gathered}
R e=\frac{\rho_{l} W R}{\mu_{l}}, \\
W e=\frac{\rho_{l} W^{2} R}{\sigma} ;
\end{gathered}
$$

$\sigma$ being the surface tension between the two phases.

- The flow rate ratio $Q=Q_{g} / Q_{l}$. 
- The swirl parameter, $S=\Gamma /(W R)$.

- The dimensionless radial velocity perturbation, $\Delta U$.

Only moderate-to-high Reynolds numbers are considered in this work since focusing can only be observed when quasi-stagnant situations (low Reynolds number, leading to bubble plug flow) are excluded. We have considered a single configuration for the the focusing liquid characterized by $R e=225$ and $W e=17.54$. For this case, we analyze the effect of parameters $Q$ and $S$ in the bubble generation when the liquid injection is perfectly axisymmetric $(\Delta U=0)$. In particular, we will focus our attention to demonstrate that the introduction of swirl reduces the size of the bubbles obtained, while the flow remains essentially axisymmetric for this case. We will also analyze the effect of non axisymmetric liquid injection, $\Delta U>0$, in the bubble generation.

\section{NUMERICAL PROCEDURE}

In order to predict the interface geometry during the time-solution, several techniques have been used, falling into one of three categories. These are: (i) interface tracking methods, including a moving mesh, ${ }^{17}$ (ii) front tracking and particle tracking schemes, ${ }^{18}$ and (iii) interface capturing methods, including volume of fluid $(\mathrm{VoF})^{19,20}$ and level set techniques ${ }^{21}$. In this work, we chose the VoF method provided by commercial solver FLUENT v 6.3 (laminar unsteady).

For the axisymmetric simulations we have discretized the domain [in the $(r, z)$-plane] into 6450 square cells. We have performed several numerical tests with smaller size mesh cells to make sure that the accuracy level is comfortably sufficient to describe the liquid flow pattern for the case under consideration $(R e=250)$ and for the range of values of the swirl parameter $(0 \leq S \leq 0.4)$. To avoid numerical diffusion of the interface when a gas stream is supplied, we have applied a dynamic mesh refinement in the gas-liquid interface region with up to two levels of refinement. For the $3 \mathrm{D}$ simulations the domain [in the $(x, y, z)$ volume] has been discretized in 336475 cube cells, where the walls of the T-junction are approximated by step functions as shown in Figure 3. Again, dynamic mesh refinement in the gas-liquid interface region has been applied.

The interface between the liquid and gas phases is tracked by solving a continuity equation 


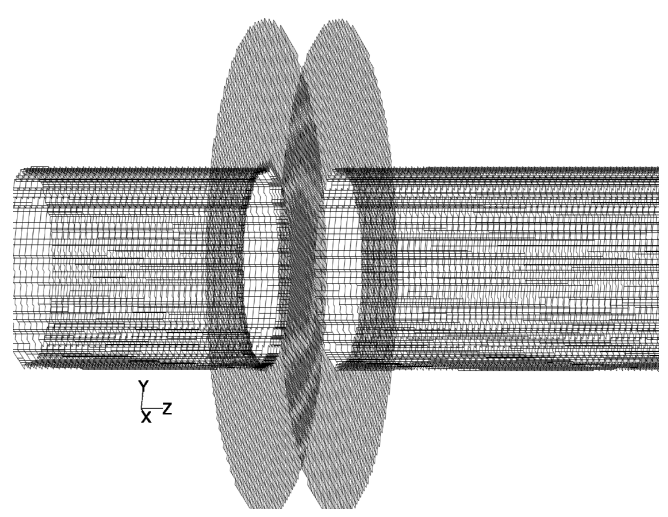

FIG. 3: Basic mesh at the wall of the $T$-junction used in the 3D simulations.

for the volume fraction of one of the phases using an explicit time-marching scheme. The rest of the equations are solved implicitly. The time steps selected were fixed and sufficiently small to ensure that the global Courant number based on the mesh cell size, the mean velocity in the cell, and the time step were always smaller than one. Regarding the spatial discretization of the equations, the third-order modified MUSCL scheme ${ }^{22}$ is used to obtain the face fluxes whenever a cell is completely immersed in a single phase. When the cell is near the interface, the Geo-Reconstruction algorithm is used. The pressure corrections are computed with the body forces weighted scheme, and the pressure-velocity coupling in a segregated solver is treated with the PISO method ${ }^{23}$.

\section{NUMERICAL RESULTS}

\section{A. Axisymmetric simulations $(\Delta U=0)$}

In the absence of swirl $(S=0)$, our numerical axisymmetric simulations show that the the bubbles are generated from a non-steady gas-liquid meniscus in a bubbling regime for any value of the flow rate quotient, $Q$. However, we will show next that, as observed in a previous work ${ }^{14}$, this unsteady meniscus can be stabilized by injecting swirl into the focusing liquid. To characterize the dripping frequency and size of the emitted bubbles, the bubble chord radius, defined as the radius of the instantaneous section of the bubble at the outlet plane, $R_{\text {bubble }}(t)$, is computed at each time step by integrating radially the gas volume fraction, $\alpha$, 

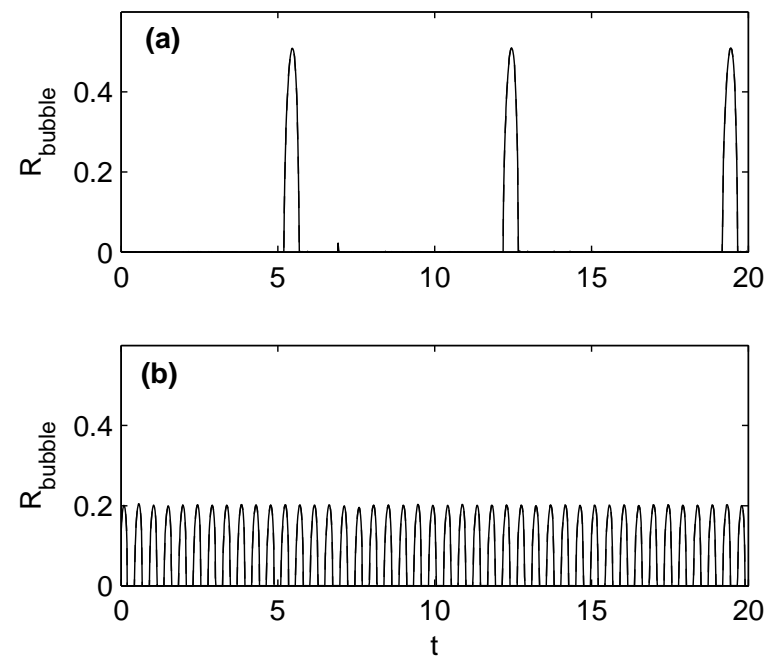

FIG. 4: Axisymmetric time dependence of the instantaneous bubble chord radius at the outlet, $R_{\text {bubble }}$, for $Q=0.0177$ and two different values of S: (a) $S=0$ and (b) $S=0.355$

at the outlet, $z=z_{\text {out }}$ :

$$
R_{\text {bubble }}(t)=\sqrt{\frac{1}{\pi} \int_{0}^{2 \pi}\left(\int_{0}^{1} \alpha\left(t, z_{\text {out }}, r, \theta\right) r \mathrm{~d} r\right) \mathrm{d} \theta} .
$$

Figure 4 shows this quantity as a function of time for $Q=0.0177$ and two different values of $S$. Note that when a bubble is crossing the outlet, the instantaneous chord radius $R_{\text {bubble }}$ is positive, while $R_{\text {bubble }}=0$ otherwise. Each local maximum, $R_{b u b b l e}^{\max }$, provides an estimate of the bubble radius at the outlet in our axisymmetric simulations. Therefore, we can deduce from the figure that for $S=0$, monodisperse bubbles whose radius is about 0.4 times the tube radius are periodically crossing the outlet. We can also see in figure 4(b) that for $S=0.355$ the exiting monodisperse bubbles are much smaller than in the absence of swirl and consequently (due to mass conservation) a dramatic rise in the frequency of generation takes place.

Figures 5(a)-5(d) show the bubble generation for $Q=0.0177$ and $S=0$, going through the stages of meniscus growth and bubble emission (unstable meniscus). The shape of the gas-liquid interface is computed in the figure as the iso-level of the gas volume fraction $\alpha=0.999$, obtained with the VoF method. In contrast, Figures 6(a)-6(d) show a stable cusplike meniscus for $Q=0.0177$ and $S=0.355$ from whose tip a steady short gas ligament breaks up into bubbles. 

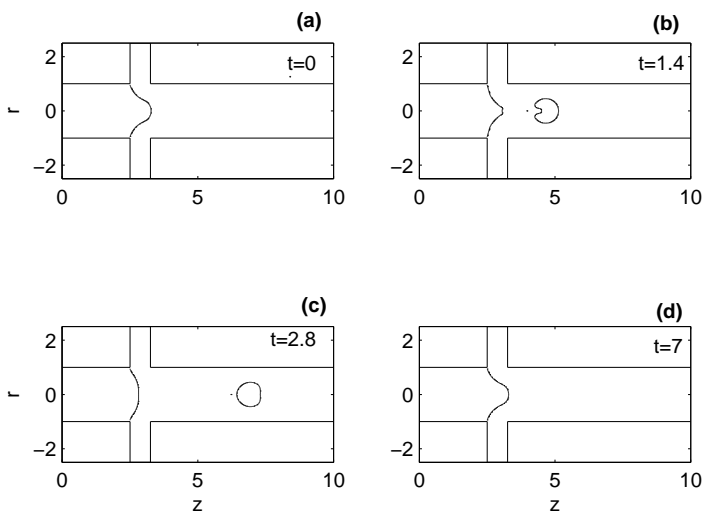

FIG. 5: A sequence of 2D-snapshots showing bubble generation for $Q=0.0177$ and $S=0$. In this case, the capillary meniscus oscillates.

A numerical study is carried out next to find the parameter region where mono-disperse bubbles are obtained. In this parameter region we characterized the size of the bubbles as a function of $Q$ and $S$. The exact volume of each bubble crossing the outlet is computed using the function

$$
Q_{\text {gas }}(t)=\int_{0}^{2 \pi}\left(\int_{0}^{1} \alpha\left(t, z_{\text {out }}, r, \theta\right) w\left(t, z_{\text {out }}, r, \theta\right) r \mathrm{~d} r\right) \mathrm{d} \theta .
$$

This quantity provides the instantaneous gas flow rate at the outlet, $Q_{\text {gas }}$, which is zero when there are no bubbles crossing that boundary, and positive otherwise. Therefore, if a bubble is crossing the exit between times $t_{1}$ and $t_{2}$, the exact volume of the bubble, $V_{\text {bubble }}$ can be computed as

$$
V_{\text {bubble }}=\int_{t_{1}}^{t_{2}} Q_{\text {gas }}(t) \mathrm{d} t
$$

With the above function and assuming a spherical geometry, $R_{b u b b l e}^{S}=\left(3 \pi V_{b u b b l e} / 4\right)^{1 / 3}$, Fig. 7 shows the bubble radius as a function of $Q$ for different swirl intensities in the parameter region where mono-disperse bubbles are obtained. Note that, as expected, in all cases the bubble size increases with $Q$. Note also that the size of bubbles generated with $S=0.355$ is almost the same as for $S=0.4$. The figure also shows the scaling law proposed by Gañán-Calvo ${ }^{24}$. The bubbles produced are slightly smaller than predicted by the scaling law in the absence of swirl, indicating a small influence of swirl on the most unstable breakup wavelength and the eventual size of the bubbles. 

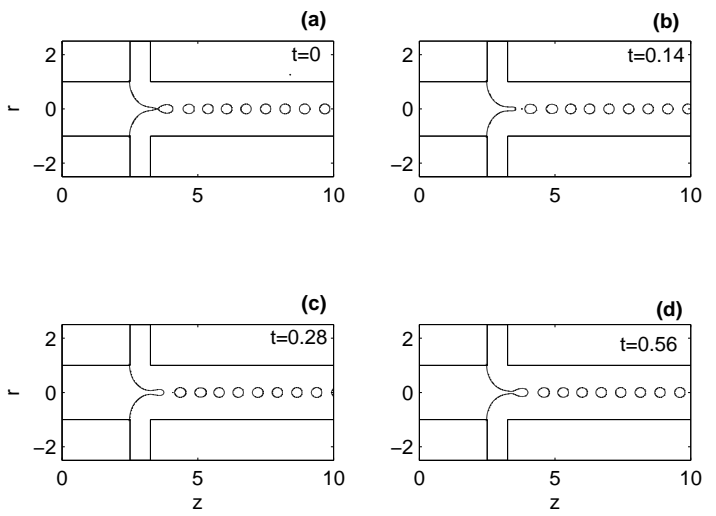

FIG. 6: A sequence of 2D-snapshots showing bubble generation for $Q=0.0177$ and $S=0.355$; the capillary meniscus is stable.

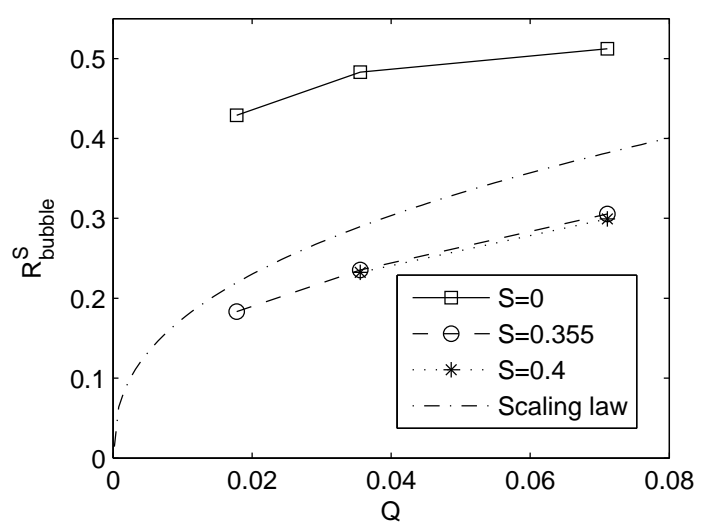

FIG. 7: Radius of the mono-disperse spherical bubbles obtained with the axisymmetric simulations as a function of $Q$ for several values of $S$.

\section{B. 3D simulations}

In this section we have carried out some 3D simulations to study the effect of a 3D finite perturbation in the liquid injection on the bubble generation. In Figure 7 it was shown that the smallest mono-disperse bubbles obtained with axisymmetric simulations are produced by a swirling flow with $S=0.355$ and $Q=0.0177$. An analysis is performed of the stability of this axisymmetrical solution when the liquid injection is perfectly axisymmetric $(\Delta U=0)$. In that case, our 3D simulations show that the flow remains essentially axisymmetric, with 


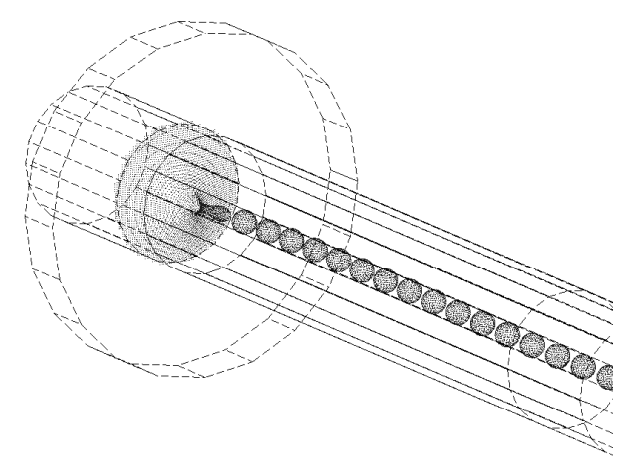

FIG. 8: A snapshot showing 3D bubble generation for $Q=0.0177, S=0.355$ and $\Delta U=0$. The dashed lines represent the tube walls.

a train of monodisperse bubbles flowing along the axis of the tube $(r=0)$. This can be seen in Figure 8, which depicts an instantaneous snapshot of the gas-liquid interface. Again, the shape of the gas-liquid interface is computed in the figure as the iso-level of the gas volume fraction $\alpha=0.999$, obtained with the VoF method. In the figure we can appreciate a stream of equal-size bubbles aligned with the tube axis, issuing from a very slightly out of axis gas nipple. This is due to the amplification of minute numerical perturbations introduced in the azimuthal direction by the space tessellation, which may resemble the effect of real irregularities in the experimental conditions. To illustrate the periodical crossing of the mono-disperse bubbles through the outlet, Figure 9 shows $R_{b u b b l e}$ (defined by equation (6)) as a function of time for this case.

The effect of changing $\Delta U$ in the bubble generation is explored next. In Figure 10 a snapshot showing 3D bubble generation for $Q=0.0177, S=0.355$ and two different values of $\Delta U$ is presented. While the trajectory of the bubbles deviates from the axis when $\Delta U$ increases, there is no appreciable change in the bubble size. As it is well known (e.g. $\left.{ }^{25}\right)$, the bubbles migrate to the region of lower pressure at the axis of the liquid vortex. Therefore, we can use the bubbles to visualize the vortex deviation from the axis of symmetry as $\Delta U$ increases. We have also found that for $S=0.333$ and $Q=0.0177$, if we increase $\Delta U$ up to $\Delta U=0.9$, bubble generation becomes quite irregular due to destabilization of the gasliquid meniscus. However, for that value of $\Delta U$, we have been able to recover a monodisperse bubble generation regime by a slight decrease of the swirl strength. In effect, in Figure 11 (a) we can see the emission of mono-disperse bubbles corresponding to a flow rate $Q=0.0177$, 


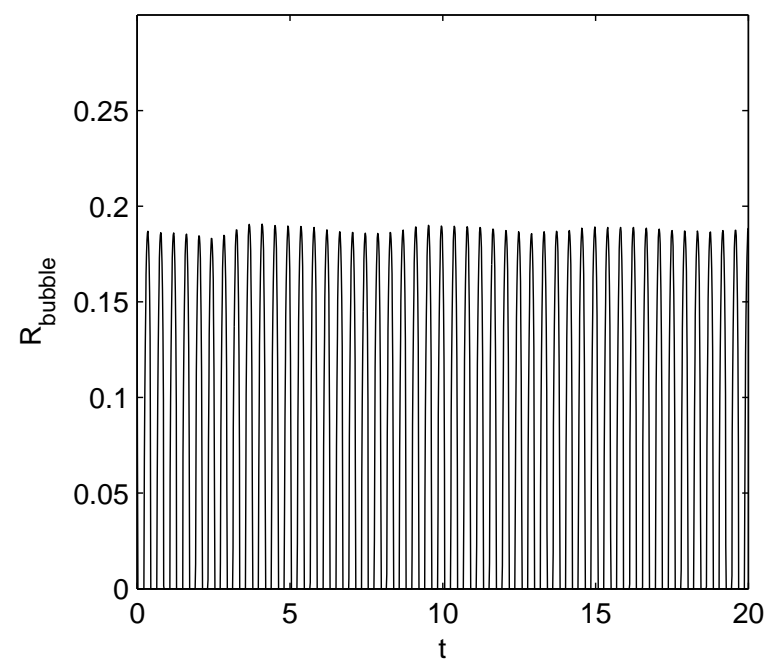

FIG. 9: 3D time dependence of the instantaneous bubble chord radius at the outlet, $R_{b u b b l e}$, for $Q=0.0177, S=0.355$ and $\Delta U=0$.

$\Delta U=0.9$ and $S=0.31$. Observe that in this case the vortex axis is close to the tube wall at some places. Figure 11 (b) shows that an increase in $Q$ leads to larger bubbles while the liquid vortex flow remains essentially the same.

\section{CONCLUSIONS}

In this work, we aim to ascertain whether a simple geometry consisting of two capillary tubes with their axes aligned and slightly offset (an axisymmetric T-junction) can lead to the production of microbubbles smaller than the inner capillary size, using water as the continuum, focusing liquid, which flows radially in the inward direction through the gap. The gas is injected through one of the capillaries and the liquid-gas two-phase flow is discharged through the other capillary (Figure 1c,d). Our hypothesis rests on the presence of a swirl in the otherwise axisymmetric and meridional liquid flow, due to the natural irregularities of the edges of the confronted capillary tubes or the presence of debris at the gap. Axisymmetric simulations indicate that the introduction of swirl induces the stabilization of the meniscus tapering into a steady neck or short gas ligament from which the bubbles issue. These results are similar to those obtained for a different flow-focusing device with different liquid configurations as proposed by Herrada and Gañán-Calvo ${ }^{14}$, and suggest that the use of a 
(a)

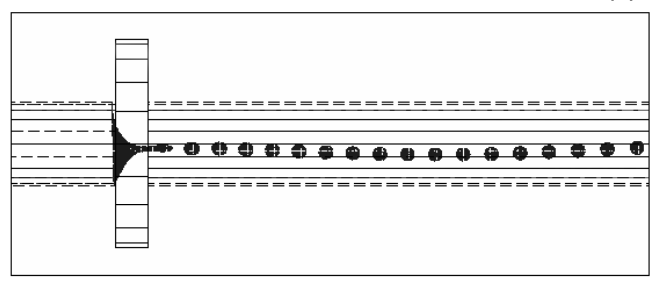

(b)

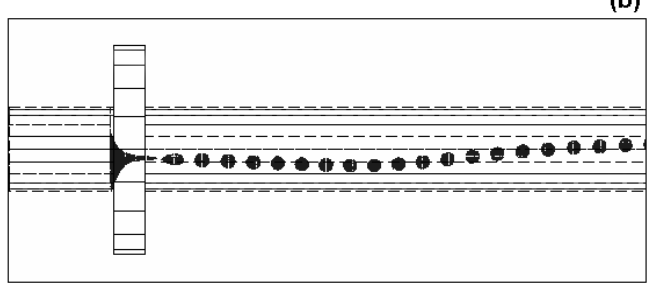

FIG. 10: A snapshot showing 3D bubble generation for $Q=0.0177, S=0.355$ and two different values of $\Delta U$; a) $\Delta U=0.333$, b) $\Delta U=0.666$. We have selected this particular view to illustrate the fact that the bubbles are not flowing along the tube axis.

(a)

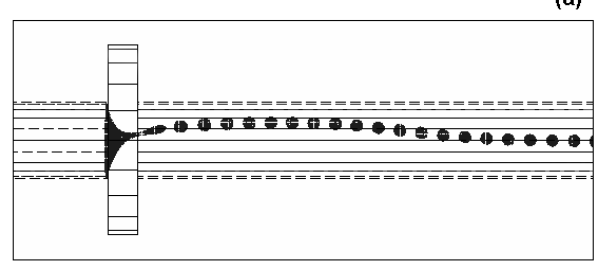

(b)

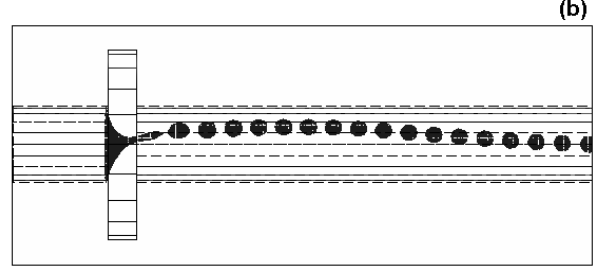

FIG. 11: A snapshot showing 3D bubble generation for $S=0.31, \Delta U=0.9$ and and two different values of $Q$; a) $Q=0.0177$, b) $Q=0.034$.

swirling liquid flow can improve bubble generation for different flow-focusing configurations.

With the help of 3D simulations we have also analyzed the effect of a non axisymmetric perturbation in the liquid injection for a specific case with $S=0.355$ and $Q=0.0177$. When 
the liquid injection is perfectly axisymmetric (azimuthal velocity perturbation $\Delta U=0$ ), the flow remains essentially axisymmetric, with monodisperse bubbles flowing along the tube axis. When $\Delta U$ is increased, a distortion of the trajectory of the bubbles becomes apparent while there is not an appreciable change in the bubble size. Only when $\Delta U$ is sufficiently large the bubble generation becomes irregular (non monodisperse) owing to destabilization of the gas-liquid meniscus. Even in that case, we were able to recover the monodisperse bubble generation regime by a slight decrease of the swirl parameter.

\section{Acknowledgments}

This research was supported by the Ministerio de Educación y Ciencia (Spain) through Grant No. DPI2007-63559. Partial support from the Junta de Andalucía through Grant No. P08-TEP-04128 is also acknowledged. Many suggestions from Prof. Pascual Riesco-Chueca are highly appreciated.

1 G. M. Whitesides. The origins and the future of microfluidics. Nature, 442:368-373, 2006.

2 G. F. Christopher and S.L. Anna. Microfluidic methods for generating continuous droplet streams. J. Physics D: Appl. Phys., 40(19):R319-R336, 2007.

3 S. Vafaei and D. Wen. Bubble formation on a submerged micronozzle. J. Colloid Interface Sci., 343(1):291 - 297, 2010.

4 T. Thorsen, R. W. Roberts, F. H. Arnold, and S. R. Quake. Dynamic pattern formation in a vesicle-generating microfluidic device. Phys. Rev. Lett., 86(18):4163-4166, 2001.

5 P. Garstecki, M. J. Fuerstman, H. A. Stone, and G. M. Whitesides. Formation of droplets and bubbles in a microfluidic t-junction - scaling and mechanism of break-up. Lab Chip, 6(3): 437-446, 2006.

6 M. De menech, P. Garstecki, F. Jousse, and H. A. Stone. Transition from squeezing to dripping in a microfluidic t-shaped junction. J. Fluid Mech., 595:141-161, 2008.

7 V. van Steijn, C. R. Kleijn, and M. T. Kreutzer. Predictive model for the size of bubbles and droplets created in microfluidic t-junctions. Lab Chip, 10:2513-2518, 2010.

8 R. Xiong, M. Bai, and J. N Chung. Formation of bubbles in a simple co-flowing micro-channel. 
J. Micromech. Microeng., 17(5):1002, 2007.

${ }^{9}$ H. N. Oguz and A. Prosperetti. Dynamics of bubble growth and detachment from a needle. $J$. Fluid Mech., 257:111-145, 1993.

10 A. M. Gañán-Calvo and J.M. Gordillo. Perfectly monodisperse microbubbling by capillary flow focusing. Phys. Rev. Lett., 87(27 I):2745011-2745014, 2001.

11 S. L. Anna, N. Bontoux, and H. Stone. Formation of dispersion using flow-focusing in microchannels. Appl. Phys. Lett., 87:364, 2003.

12 P. Garstecki, I. Gitlin, W. Diluzio, G.M. Whitesides, E. Kumacheva, and H.A. Stone. Formation of monodisperse bubbles in a microfluidic flow-focusing device. Appl. Phys. Lett., 85(13):2649$2651,2004$.

13 J. M. Gordillo, Z. Cheng, A. M. Ganan-Calvo, M. Mßrquez, and D. A. Weitz. A new device for the generation of microbubbles. Phys. Fluids, 16(8):2828-2834, 2004.

14 M. A. Herrada and A. M. Gañán-Calvo. Swirl flow focusing: A novel procedure for the massive production of monodisperse microbubbles. Phys. Fluids, 21:042003, 2009.

15 M. A. Herrada and R. Fernandez-Feria. On the development of three-dimensional vortex breakdown in cylindrical regions. Phys. Fluids, 18(8), 2006.

16 A. M. Gañán-Calvo. Universal scaling of microbubble size produced by capillary flow focusing. Bull. Am. Phys. Soc., 48(10):224, 2003.

17 D. B. Kothe and W. J. Rider. A comparison of interface tracking methods. Fluid Dynamics, pages 19-22, March 1995.

18 S. Ozen Unverdi and G. Tryggvason. A front-tracking method for viscous, incompressible, multi-fluid flows. J. Comput. Phys., 100(1):25-37, 1992.

19 D. J. E. Harvie and D. F. Fletcher. A new volume of fluid advection algorithm: the stream scheme. J. Comput. Phys., 162(1):1-32, 2000.

20 E. Aulisa, S. Manservisi, and R. Scardovelli. A mixed markers and volume-of-fluid method for the reconstruction and advection of interfaces in two-phase and free-boundary flows. J. Comput. Phys., 188(2):611-639, 2003.

21 S. Tanguy and A. Berlemont. Application of a level set method for simulation of droplet collisions. Int. J. Multiphase Flow, 31:1015-1035, 2005.

22 B. Van Leer. Toward the ultimate conservative difference scheme. iv. a second order sequel to godunov's method. J. Comput. Phys., 32:101-136, 1979. 
23 R. I. Issa. Solution of implicitly discretized fluid flow equations by operator splitting. J. Comput. Phys., 62:40-65, 1986.

24 A. M. Gañán-Calvo. Perfectly monodisperse microbubbling by capillary flow focusing: An alternate physical description and universal scaling. Phys. Rev. E, 69:027301, 2004.

25 D. Bonn, Y. Couder, P.H.J. van Dam, and S. Douady. From small scales to large scales in three-dimensional turbulence: the effect of diluted polymers, Phys. Rev. E 47, . Phys. Rev. E, 47:R28-R31, 1993. 